Association for Information Systems AIS Electronic Library (AISeL)

Wirtschaftsinformatik Proceedings 2001

Wirtschaftsinformatik

September 2001

\title{
Konzeption eines Balanced Scorecard Systems zur Planung und Steuerung im Krankenhaus
}

Hans Czap

UniversitätTrier, cz@wiinfo.uni-trier.de

Stefan Winkel

UniversitätTrier, sw@wiinfo.uni-trier.de

Follow this and additional works at: http://aisel.aisnet.org/wi2001

\section{Recommended Citation}

Czap, Hans and Winkel, Stefan, "Konzeption eines Balanced Scorecard Systems zur Planung und Steuerung im Krankenhaus" (2001). Wirtschaftsinformatik Proceedings 2001. 63.

http://aisel.aisnet.org/wi2001/63

This material is brought to you by the Wirtschaftsinformatik at AIS Electronic Library (AISeL). It has been accepted for inclusion in Wirtschaftsinformatik Proceedings 2001 by an authorized administrator of AIS Electronic Library (AISeL). For more information, please contact elibrary@aisnet.org. 
In: Buhl, Hans Ulrich, u.a. (Hg.) 2001. Information Age Economy; 5. Internationale Tagung Wirtschaftsinformatik 2001. Heidelberg: Physica-Verlag

ISBN: 3-7908-1427-X

(C) Physica-Verlag Heidelberg 2001 


\title{
Konzeption eines Balanced Scorecard Systems zur Planung und Steuerung im Krankenhaus
}

\author{
Hans Czap, Stefan Winkel \\ Universität Trier
}

Zusammenfassung: Krankenhäuser befinden sich in einer immer kritischer werdenden Umweltsituation, die eine große Herausforderung für das Management darstellt. Die Balanced Scorecard BSC ist ein System, das eine Lösung für schwieriger werdende Umweltsituationen verspricht. Ein kurzfristig wirkender, instrumentaler Charakter der BSC, der sich in einem Kennziffernsystem ausdrückt, und eine langfristige, strategische und verhaltensändernd wirkende Komponente werden identifiziert.

Ausschnitte eines Kennziffernsystems für Krankenhäuser zum Zwecke der Planung und Steuerung werden vorgestellt und Anforderungen an die technische Realisierung abgeleitet. Das grundlegende Datenmodell wird in Ausschnitten erläutert.

Schlüsselworte: Balanced Scorecard, Kennziffernsystem, Data Warehouse, operative Planung

\section{Krankenhausmanagement in einem dynamischen Umfeld}

Das Gesundheitswesen im allgemeinen, speziell aber Krankenhäuser sehen sich seit einigen Jahren mit vielfältigen Herausforderungen konfrontiert. Die Umwelt von Krankenhäusern erweist sich in zunehmendem Maße als dynamisch. Es fehlt an Kontinuität bzw. Stabilität. Vielfältige Gründe erklären dieses Phänomen.

An herausragender Stelle ist der medizinische Fortschritt zu nennen und die Notwendigkeit diesem beständig Rechnung zu tragen. Dies bedingt aus Sicht der Krankenhausverwaltung, dass unter anderem der Weiterbildung der Mitarbeiter besondere Aufmerksamkeit gewidmet werden muss. Aber auch der Bedarf an laufenden Investitionen erfährt vor diesem Hintergrund eine besondere Relevanz.

Gleichfalls an herausragender Stelle ist die demographische Entwicklung der Bevölkerung in Deutschland zu sehen. Die Menschen leben heute länger als in früheren Jahren, sie fragen damit länger und intensiver nach Leistungen des Gesundheitswesens. 
Auch steigt seit einiger Zeit der Anteil der Bevölkerung im Rentenalter rapide an, so dass von einer Veralterung der Gesellschaft gesprochen wird. Die Kosten des Gesundheitswesens müssen im Bereich der gesetzlichen Krankenkassen deswegen auf tendenziell immer weniger Beitragszahler verteilt werden. Dies rief den Gesetz- bzw. Verordnungsgeber auf den Plan, der mit einer Flut von Gesetzen zur Kostendämpfung beitragen möchte (vgl. [Ned 01], S. 82). Daraus resultieren umfangreiche Anforderungen an das Management von Krankenhäusern, auf die weiter unten eingegangen wird.

Geändert hat sich die Einstellung der Patienten. Der Patient wird mündiger, er ist aufgeklärter und verlangt verstärkt Auskunft und Rechenschaft über durchgeführte bzw. geplante Maßnahmen. Die Sensibilisierung über den Preis von Gesundheitsleistungen, aber auch der leichtere Zugang zu Informationen im Gesundheitsbereich (einschlägige Literatur, Internet, Bildung von Interessensgemeinschaften bei chronischen Erkrankungen mit entsprechendem Informationsaustausch) verändert das Arzt-Patientenverhältnis. Der Patient will Mitsprache und Einbeziehung bei der Chancen- und Risikenabwägung vorgesehener diagnostischer und therapeutischer Maßnahmen.

Die Krankenhausfinanzierung befindet sich in einem drastischen Umbruch und bedingt erhöhte Anforderungen an Führung und Steuerung im Krankenhaus. Die derzeit praktizierte Deckelung der Krankenhausbudgets hat die systemwidrige Konsequenz, dass Mengen von Krankheitsfällen pro Jahr für das Krankenhaus vereinbart und entsprechend angesteuert werden, wobei Abweichungen finanzielle Einbußen zur Folge haben. Erwartet wird in absehbarer Zeit der Übergang zur Monistik, d.h. zukünftig werden auch die Investitionen aus dem erwirtschafteten Entgelt getätigt werden müssen. Ab Januar 2003 ist die Einführung von umfassenden Fallpauschalen (bis auf die Leistungen der Psychiatrie) geplant. Diese sogenannten Diagnosis Related Groups DRG's stellen ein Preissystem dar, das pro Fall, also pro Patient und Erkrankung, ein festes Entgelt vorsieht (vgl. [Böck 01], S.49ff). Dieses Entgelt wird der Idee nach und nach Ablauf einer Übergangsfrist unabhängig vom Krankenhaus festgesetzt. Das kostengünstigere Krankenhaus kann sich folglich Vorteile erhoffen, während das ineffizient wirtschaftende Krankenhaus im Extremfall zu schließen ist.

Ashby's Gesetz der beschränkten Varietät besagt, dass die Zunahme der auf ein System aus der Systemumwelt wirkenden Einflussfaktoren nach Art und Anzahl (Störgrößen) zwangsläufig eine Steigerung der systeminhärenten Komplexität zur Folge hat, um das System in seiner Existenz zu erhalten. Übertragen auf das System Krankenhaus bedeutet das, dass die Entscheidungsträger des Krankenhauses diesen vielfältigen Herausforderungen nur durch differenzierte Maßnahmen begegnen können. Die Komplexität nimmt zu und damit die Anforderungen an ein adäquates Informations- und Managementsystem.

Verstärkt sucht die Krankenhausverwaltung nach geeigneten Methoden und Instrumenten, um diesen vielfältigen Herausforderungen zu begegnen. Die Balanced 
Scorecard (BSC) von Kaplan und Norton [Kap 97] erfreut sich in diesem Zusammenhang einer besonderen Aufmerksamkeit, da sie das kurzfristige operative Controlling mit einem strategisch ausgerichteten, eher längerfristig wirksamen Controlling verbindet.

Anhand einer konkreten Umsetzungsstudie will dieser Beitrag den Nutzen bzw. das Nutzenpotential eines BSC-Systems aufzeigen. Angesichts des momentanen Handlungsdrucks, dem sich das Krankenhausmanagement ausgesetzt sieht, konzentriert sich die vorgelegte Studie eher auf kurzfristig wirksame Maßnahmen.

\section{Die Idee der Balanced Scorecard}

Wie alle erfolgreichen Managementideen der jüngeren Zeit besticht die Balanced Scorecard durch ein einfaches Grundprinzip. Ein Unternehmen, das sich in einer dynamischen Umwelt erfolgreich behaupten will, muss sich gleichzeitig an mehreren Führungsgrößen orientieren. Auf eine kurze Formel gebracht lautet das Gebot: Mannigfaltigkeit statt Einfältigkeit. Es reicht nicht, ein gutes Produkt zu haben, aber die Kundenbeziehungen zu vernachlässigen oder den kurzfristigen Umsatz zu maximieren auf Kosten der Zukunftsfähigkeit, beispielsweise unter Vernachlässigung von Forschung und Entwicklung.

Mehrere Erfolgsdimensionen sind also gleichzeitig zu betrachten und in einem ausgewogenen Verhältnis, „balanciert“, zu halten. Um Erfolg oder Misserfolg feststellen zu können, müssen diese Erfolgsdimensionen messbar sein, oder zumindest in akzeptabler Weise durch Zahlen erfasst werden können. Die „Scorecard“" ist vergleichbar einem Punktekonto, das den relativen Erfolg misst.

Bezugsgrößen können Vergangenheitsdaten sein, soweit eine stetige Entwicklung gegeben ist und als Erfolgsausweis sinnvoll erscheint. In Bereichen bzw. bei Größen, die Strukturbrüchen und Diskontinuitäten ausgesetzt sind, wie dies teilweise im Krankenhaus, beispielsweise bei Abrechnungsgegebenheiten aufgrund sich ständig ändernder Vorschriften der Fall ist, muss ergänzend mit Plan- bzw. Sollzahlen gearbeitet werden.

Nicht ganz so einfach entscheidbar ist die Frage, welche Erfolgsdimensionen für ein BSC-System auszuwählen sind. In ihrem Werk zur Balanced Scorecard [Kap 97] beschränken sich Kaplan und Norton auf vier Perspektiven mit denen der Status des Unternehmens abzubilden ist, nämlich die finanzwirtschaftliche Perspektive, die Kundenperspektive, die internen Prozesse und die Lern- und Entwicklungsperspektive.

Dabei ist nicht so sehr die konkrete Auswahl der Perspektiven entscheidend. Wichtig sind vielmehr folgende Kriterien, die für Auswahl und Umsetzung Orientierungshilfe geben: 


\section{Vollständigkeit und Übersichtlichkeit}

Vollständigkeit meint in diesem Zusammenhang, dass alle wichtigen Unternehmensbereiche zu erfassen sind. Sowohl kurzfristige Erfolgsgrößen des operativen Geschäfts, als auch wichtige Maßgrößen zur Sicherung künftiger Erfolge. Ersteres spricht die klassischen Kennziffern des finanzwirtschaftlichen Bereichs an, letzteres bezieht sich mehr auf den Wissenstand der Mitarbeiter und dessen permanente Pflege, auf Investitionen in Forschung und Entwicklung, auf ein systematisches Customer Relationship Management etc.

Übersichtlichkeit bedeutet Komprimierung auf einige wenige Größen. Je nach Unternehmensebene wird man deswegen mit hierarchisch verdichteten Kennziffern arbeiten.

Übersichtlichkeit bedeutet aber auch, dass nur einige wenige Perspektiven auszuwählen sind. Damit stellt Übersichtlichkeit einen Widerspruch zur Vollständigkeit dar, die tendenziell möglichst viele Kriterien zu erfassen sucht. Beide Ziele stehen in Konkurrenz zueinander. Sie sind folglich ausgewogen, balanciert, zu gestalten.

\section{Authentizität: Integration von Vision, Strategie und konkreten Maßnahmen}

Dauerhafter Erfolg kann sich nur einstellen, wenn alle Abteilungen und alle Ebenen einer Unternehmung bzw. eines Krankenhauses geschlossen ein gemeinsames Ziel verfolgen. Dazu ist eine Vision hilfreich, also beispielsweise eine Vorgabe der Art „Im Jahr 2010 wollen wir das Krankenhaus mit der besten Reputation in der allgemeinen Grundversorgung sein!“ oder „In fünf Jahren sind wir überregional führend in der Mikrochirurgie!“”. Authentizität bedeutet in diesem Zusammenhang, dass derartige Visionen von der Gesamtbelegschaft getragen werden müssen. Dies bedingt nach Beschluss in der Geschäftsleitung eine Selbstverpflichtung aller Führungskräfte, eine überzeugende Vermittlung auf die tieferen Ebenen des Krankenhauses und die konsistente Umsetzung in strategische Ziele und daraus abgeleitet konkrete Maßnahmen. Denn ein visionäres Ziel, wie das Erstreben einer hohen Reputation oder überragender Qualität umfasst letztlich alle Abteilungen und Stellen eines Krankenhauses.

Der Erfolg eines derartigen Programms kann unseres Erachtens nicht befohlen werden. Über Befehl und Gehorsam wird allenfalls ein halbherziges Mitmachen bewirkt, kein freiwilliges Engagement aufgrund von Überzeugung.

Die in deutschen Krankenhäusern typischerweise vorhandenen ausgeprägten hierarchischen Strukturen vertragen sich deswegen nur schlecht mit Idee und Wesen der BSC. Angestrebt werden muss eine andere Krankenhauskultur. Schon allein aufgrund der Komplexität der Krankenhausprozesse und des hohen Risikos, dem alle Beteiligten ausgesetzt sind, brauchen wir den mitdenkenden Mitarbeiter, der, natürlich im Rahmen der gesetzlichen Vorschriften, eigenverantwortlich handelt. Dies bedeutet Beteiligung von Untergebenen an der Entscheidungsfindung, verstärkte Delegation von Aufgaben, die von den Mitarbeitern selbständig erledigt werden können und die Bereitschaft bei den Vorgesetzten Fehler von Untergebe- 
nen zu akzeptieren und zu verstehen, dass jegliches Lernen nur möglich ist, wenn Fehler zugelassen werden.

Die Ausführungen des letzten Absatzes zeigen, dass die BSC nicht als ausschließlich zeitlich befristetes Projekt missverstanden werden darf.

Es geht um zwei Perspektiven. Einerseits um eine kurzfristige, instrumentale, bei der die Einführung geeigneter Kennziffern zur Unterstützung des operativen Geschäfts erfolgt. Andererseits verfolgt die BSC einen radikalen, am grundsätzlichen orientierten Ansatz, der zu einer geänderten Krankenhauskultur führt. Erstere Perspektive hat mehr den Charakter einer Investition, deren Kosten und Nutzen in Geldeinheiten ausgedrückt werden kann. Letztere fordert ein anderes Denken im Krankenhaus und kann nur Ergebnis einer längeren Diskussion sein.

Nachfolgend wird ein Fokus auf den kurzfristigen instrumentalen Charakter der BSC gesetzt.

\section{Planung und Steuerung durch finanzwirtschaftliche Kennziffern}

Die Krankenhausbuchführungsverordnung sowie diverse weitere Vorschriften, wie beispielsweise $\S 301$ SGB V, bedingen die Sammlung einer Vielzahl von Daten, deren systematische Auswertung längst überfällig ist und in der Mehrheit der Krankenhäuser bislang noch nicht hinreichend erfolgte. Um das vorhandene Potential anzudeuten werden zwei Teilbereiche betrachtet. Zum einen das Teilsystem „Finanzwirtschaftliche Kennziffern im Krankenhaus“ (Abbildung 1), das auf den derzeitigen gesetzlichen Grundlagen beruht und am Zentrum für Gesundheitsökonomie der Universität Trier entwickelt wurde (im Bezug auf Krankenkassen vgl. [Haen 01]). Zum anderen zeigen wir, wie die aktuell hochrelevante Einführung des Australischen DRG-Systems durch eine Kennziffernsystem unterstützt werden kann.

\subsection{Kennziffern basierend auf Bilanz und GuV}

Entsprechend $\S 8$ KHBV beruht das System auf den Primärdaten der Gewinn- und Verlustrechnung (GuV) sowie der Bilanz und zielt darauf ab, als Steuerungs- und Kontrollinstrument auf der Ebene eines Krankenhauses und eines Krankenhausträgers eingesetzt zu werden. Größere Häuser müssen Bilanz und GuV veröffentlichen. Dies ermöglicht einen Vergleich bzw. ein Benchmarking mit anderen Häusern.

Beispielhaft werden die Erlösquoten näher betrachtet. Erlösquoten geben den Anteil der einzelnen Erlösarten am Gesamterlös wieder. Für eine leistungsbezogene 
ABC-Analyse bzw. bei sich abzeichnenden Preisänderungen, Änderungen der Gewinnspannen oder Marktpotentiale ist eine Quotenbildung als steuerungsrelevante Maßnahme unerlässlich.

Ex-post betrachtet erhöhen Erlösquoten u.a. die Vergleichbarkeit zwischen Krankenhäusern und Perioden. Auch führen derartige Detailinformationen zu einer Relativierung des „Return on Investments“ (ROI), da die Höhe des ROI wesentlich von der Erlösstruktur abhängt.

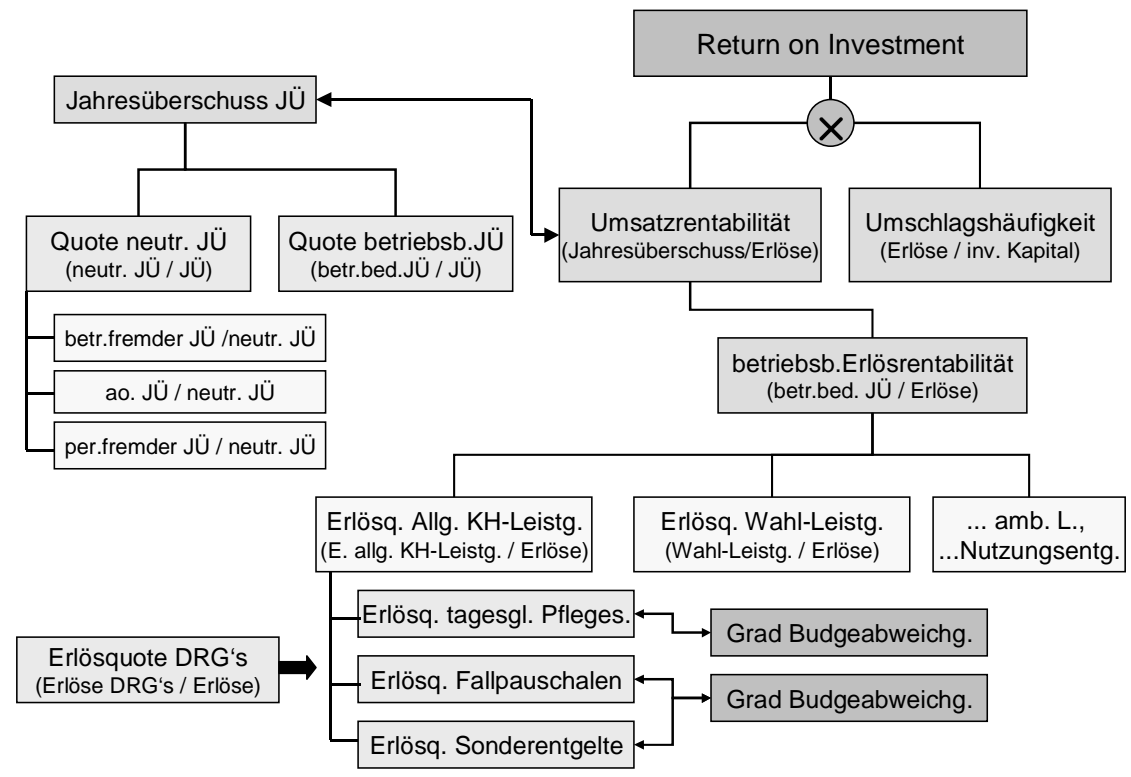

Abbildung 1: Finanzwirtschaftliche Kennziffern im Krankenhaus

Ex-ante betrachtet erlauben Erlösquoten Simulationen oder Sensibilitätsanalysen, mit deren Hilfe der zukünftige Erfolg zusichern oder auszubauen ist. Besonders in Verbindung mit Kennzahlen der Komponente „Kosten- und Leistungsrechnung“ können basierend auf den Gewinnen je Leistungsart die Auswirkungen einer schwerpunktmäßigen Verlagerung innerhalb der verschiedenen Erlösquoten auf den ROI abgebildet werden.

Die Summe aller budgetierten Krankenhausleistungen dient zur Bildung der Kennzahl „Erlösquote der allgemeinen Krankenhausleistungen“. Diese Kennzahl wird zur differenzierteren Betrachtung bzw. Abgrenzung der verschiedenen budgetierten Erlösarten aufgesplittet. Mittels der Aufsplittung und einer entsprechenden Abweichungs- bzw. Schwachstellenanalyse sind Ursachen negativer Tendenzen zu lokalisieren. 
Zur Erläuterung sei eine Erlösquoten des „Kennzahlenzweiges“ der Abbildung 1 näher betrachtet, nämlich die „Erlösquote bei angenommener Punktlandung hinsichtlich tagesgleicher Pflegepflegesätze“"

In der Regel genießen Unternehmen mit einem höheren Erlös ein wirtschaftlich kompetenteres Ansehen als vergleichbare Unternehmen mit niedrigerem Erlös. Bei Krankenhäuser ist dies u.a. deshalb differenzierter zu sehen, weil die Erlöse aus allgemeinen Krankenhausleistungen budgetiert sind. Ein mengen- und damit betragsmäßiges Abweichen von den vereinbarten Budgets ist mit Sanktionen in Form von Erlösschmälerungen verbunden. Aus Sicht der Krankenhäuser wird deswegen eine budgetbezogene „Punktlandung“ angestrebt.

Die Budgets stellen somit grundsätzlich eine Erlösgrenze dar, die Krankenhäuser mit höherem Erlöspotential gegenüber Krankenhäusern mit geringerem Erlöspotential ,ausbremsen“. Dies ist ein Grund dafür, das neben der o.g. Erlösquote die nachfolgenden Kennzahlen „Erlösquote bei angenommener Punktlandung“ und „Grad der Budgetabweichung“" zu betrachten sind.

Die „Erlösquote bei angenommener Punktlandung hinsichtlich tagesgleicher Pflegepflegesätze“ gibt den Anteil des vereinbarten Budget für tagesgleiche Pflegesätze am Gesamterlös des Krankenhauses wieder. Je höher dieser Anteil ist, desto stärker ist das Krankenhaus von der Vergütungshöhe der budgetierten Leistungen abhängig.

Darüber hinaus kann diese Kennzahl zur Bestimmung verwendet werden, inwieweit zu einem bestimmten Zeitpunkt eine Abweichung vom angestrebten Ziel „Punktlandung“"droht.

Die Kennzahl „Grad der Budgetabweichung“ ermittelt sich als Quotient aus „Erlöse aus tagesgleichen Pflegesätzen“ und „Vereinbartes Budget für tagesgleiche Pflegesätze“. Sie zeigt an, inwieweit zu einem bestimmten Zeitpunkt entweder vom vereinbarten Budget abgewichen wird oder mit einer bestimmten Abweichung zu rechnen ist.

Dabei befindet sich im Zähler die Erlöshöhe, welche das Krankenhaus ohne Budgetierung bekommen hätte.

$\mathrm{Zu}$ berücksichtigen ist, das die Entscheidungsträger unter Umständen aus strategischen Gründen um einen bestimmten Betrag vom vereinbarten Budget abweichen wollen und damit eine inoffizielle Punktlandung anvisieren. Diese Punktlandung dient dann als Ausgangsbasis für zukünftige Budgetverhandlungen.

\subsection{Kennziffern zur Steuerung und Kontrolle der Erlöse nach Einführung der Fallpauschalen AR-DRG's}

§ 301 SGB V (Sozialgesetzbuch V) verpflichtet die Krankenhäuser den Krankenkassen eine Vielzahl von Daten maschinenlesbar zu übermitteln. Zusätzlich werden zur Ermittlung der ab dem Jahr 2003 vorgesehenen Diagnosis Related Groups 
DRG's das Gewicht bei Neugeborenen sowie die Nebendiagnosen benötigt. Die AR-DRG's klassifizieren zunächst in die Haupterkrankungsgruppen, die sogenannten Major Desease Categories (MDC's) und innerhalb dieser entsprechend der Hauptdiagnose in die Behandlungsgruppe DRG.

Das Australische System erlaubt weiter eine Unterteilung in unterschiedliche Schweregrade, Untergruppen A bis D. Die Erstattungsunterschiede bei unterschiedlichen Schweregraden haben teilweise erhebliche Größenordnung. Beispielsweise beträgt bei einer angenommenen Basisrate von DM 3015,- das Entgelt bei der DRG L63A (Kidney and Urinary Tract Infections, Age>69 W Catastrophic CC) DM 6.035,- , während das Entgelt der gleichen DRG bei geringstem Schweregrad (bei L63 gibt es nur die Untergruppen A bis C) L63C (Kidney and Urinary Tract Infections, Age < 70) DM 2.348,75 beträgt.

Wesentlichen Einfluss auf die Einstufung in höhere Schweregrade haben die Nebendiagnosen. Deren vollständige und systematische Erfassung liegt im unmittelbaren Interesse der Krankenhausverwaltung.

Im folgenden Beispiel zeigen wir einen Ausschnitt aus einem Kennziffernsystem, das die Auswirkungen der Vergütung nach dem AR-DRG-System (das deutsche DRG-System ist zum Zeitpunkt der Erstellung dieser Schrift noch nicht verfügbar) im Vergleich zur herkömmlichen Abrechnung nach Bundespflegesatzverordnung BPflV aufzeigt.

Als Input für eine Gruppierung in DRG's werden folgende Daten benötigt: Fallnummer bzw. laufende Nummer, Zugeordnete Abteilung (entlassende Abteilung), Entlassungsstatus, Alter (Jahr oder bei neugeborenen Tage), Geschlecht, Gewicht (bei Neugeborenen), Verweildauer, Hauptdiagnose, Nebendiagnosen, Haupteingriff, Zusatzeingriffe, Verlegung intern (Anzahl). Diese Daten lassen eine Auswertung der erzielten Abrechnungshöhe pro DRG, MDC, Abteilung und für das gesamte Krankenhaus zu.

In Tabelle 1 wurden einige wichtige Kennziffern zusammengestellt, die sich aus der Analyse der Abrechnungsdaten einer Krankenhausabteilung für ein Quartal ergaben.

Zeile (1) entspricht der derzeitigen australischen Basisrate. Zeilen (2) - (5) ergeben sich durch Auszählung der Grunddaten. Aus Zeile 6 errechnet sich die durchschnittliche Verweildauer, die mit einem Wert von 10,22 besser ist als der 


\begin{tabular}{|c|l|r|}
\hline \multicolumn{3}{|c|}{ Abteilung: Innere Medizin } \\
\hline$(1)$ & Zeitraum: 1.1.2001 -31.3.01 & \\
\hline$(2)$ & angenommene Basisrate & $3.015,00 \mathrm{DM}$ \\
\hline$(3)$ & Anzahl Fälle & 482 \\
\hline$(4)$ & $\varnothing$ Alter & 65,4 Jahre \\
\hline$(5)$ & Anzahl Nebendiagnosen & 439 \\
\hline$(6)=(3) /(2)$ & $\varnothing$ Verweildauer & 10,22 \\
\hline$(7)=(5) /(2)$ & $\varnothing$ Anzahl Nebendiagnosen & 0,91 \\
\hline$(8)$ & Anzahl Tage gemäß DRG's & 1977,74 \\
\hline$(9)=(8) /(2)$ & $\varnothing$ Verweildauer gemäß DRG's & 4,10 \\
\hline$(10)$ & Case Mix & 467,24 \\
\hline$(11)=(10) /(2)$ & Case Mix Index CMI & 0,97 \\
\hline$(12)$ & PCCL (Pat. Clinical Complexity Level) & 0,73 \\
\hline$(13)$ & Erlös gemäß BPflV & $2.181 .457,10 \mathrm{DM}$ \\
\hline$(14)=(1) *(10)$ & künftiger Erlös gemäß DRG's & $1.408 .728,60 \mathrm{DM}$ \\
\hline$(15)=(13)-$ & Erlösschmälerung, absolut & $772.728,50 \mathrm{DM}$ \\
$(14)$ & & $35,42 \%$ \\
\hline$(16)=(15) /(13)$ & Erlösschmälerung in \% & $4.668,82 \mathrm{DM}$ \\
\hline$(17)=(13) /(10)$ & Erford. Basisrate für Kostenneutralität \\
\hline
\end{tabular}

Tabelle 1: Kennziffern zur Umstellung auf DRG's

bundesdeutsche Wert in Höhe von 11,3 [Böck 01, S. 53]. Trotzdem ergibt sich verglichen mit dem australischen Wert in Höhe von 4,1 (Zeile (9)) eine erhebliche Diskrepanz. Den einzelnen DRG's liegen durchschnittliche Verweildauern zugrunde. Diese bestimmen den für die Berechnung von (9) benötigten Wert aus Zeile (8).

Der Case Mix, Zeile (10), bestimmt sich als Summe der Häufigkeiten der einzelnen DRG's, gewichtet mit dem sogenannten Case Wert cw. Der Case Wert multipliziert mit der Basisrate gibt den Erlös pro DRG und Fall wieder.

Ein erster kritischer Hinweis ergibt sich aus Zeile (7), der durchschnittlichen Anzahl an Nebendiagnosen. Verglichen mit dem mittleren Alter der Patienten von 65,4 Jahren, wäre mit einer wesentliche höheren Anzahl an Nebendiagnosen zu rechnen. In die gleiche Richtung zielen der Case Mix Index CMI, Zeile (11) sowie der PCCL (12), die beide kleiner als 1 sind.

Der CMI gewichtet mit der Basisrate gibt den mittleren Erlös pro Patient an. Ein CMI-Wert ungefähr 1,5 gilt als angemessen. Ein CMI kleiner als 1 besagt, dass im Schnitt pro Patient weniger als die Basisrate erlöst wird. 
Der Patient Clinical Complexity Level PCCL nimmt Werte zwischen 0 und 4 an. Null besagt, dass keine Komplikationen oder Komorbidität (Complication and Comorbidity Level CCL $=0$ ) gegeben sind, PCCL $=4$ bedeutet schwere bis katastrophale Komplikationen $(\mathrm{CCL}=4)$. Der Wert PCCL $=0,73$ zeigt an, dass das Patientengut, so wie es dokumentiert ist, überdurchschnittlich gesunde Patienten enthält. Dies steht im Widerspruch zum mittleren Alter der Patienten. Mit anderen Worten, die Dokumentation an Nebendiagnosen, die wesentlich für die Ermittlung höherer Komplexitätswerte PCCL's ist, ist offensichtlich ungenügend. Dies drückt sich letztlich dann auch in der Erlösschmälerung (15) aus.

Der Gesetzgeber plant in der Anfangszeit die Einführung der DRG's erlösneutral für die Krankenhäuser umzusetzen. Für die hier untersuchte Abteilung hieße das, dass anstelle der Basisrate in Höhe von DM 3.015,- die Basisrate in Höhe von DM $4.668,82$ vereinbart werden müsste.

In den Ausführungen dieses Kapitels war jeweils eine feste Bezugsbasis bei den erwähnten Kennziffern unterstellt. Dies ist jedoch keinesfalls zwingend. Vielmehr macht es in vielen Fällen Sinn, mehrere, aufeinander aufbauende Bezugsbasen zu wählen, also beispielsweise einzelne Abteilungen, Kostenstellen, Hauptdiagnosegruppen (Major Disease Categories MDC's), Fallpauschalen etc. Dieser Gedanke findet sich in dem folgenden Abschnitt über technische Aspekte wieder, wenn von hierarchischen Zusammenfassungen bzw. Verdichtungen entlang ausgewählter Dimensionen im OLAP-Würfel gesprochen wird.

\section{Technische Aspekte}

\subsection{Anforderungen an die DV-Basis}

Moderne Planungs- und Steuerungsinstrumente kommen ohne Vergleichsdaten nicht aus. Sie benötigen in aller Regel Daten früherer Rechnungsperioden um Trends $\mathrm{zu}$ ermitteln, Fortschreibungen $\mathrm{zu}$ ermöglichen oder auch typische Schwankungen aufzuzeigen und damit Abweichungen als normal oder außerordentlich klassifizieren zu können. Gleiches gilt für die in diesem Beitrag exemplarisch betrachteten Kennziffern des finanzwirtschaftlichen Bereichs.

Eine Sammlung historischer Daten sollte physisch getrennt sein von den Daten des operativen Geschäfts. Diese Forderung begründet sich primär aus der unterschiedlichen Abfragecharakteristik eines „online analytical processing“ (OLAP) gegenüber den transaktionsorientierten Datenbanken, die zur Unterstützung der laufenden Unternehmensprozesse benötigt werden (vgl. z.B. [Vos 99] S.672). Sie begründet sich aber auch aus dem riesigen Speicherbedarf, typischerweise in Terabytes ausgedrückt, vergangenheitsorientierter Datensammlungen. Als zentrale technische Basis bietet sich deswegen der Einsatz eines Data Warehouse an (vgl. [ThiKR97], S223ff). 
Die üblichen OLAP-Techniken ([Bau 01], S. 104 ff), die in Verbindung mit einem Data Warehouse angeboten werden, also Auswahl der anzuzeigenden Dimensionen, hierarchische Zusammenfassungen bzw. Verdichtungen entlang der Dimensionen, Slice (Heraustrennen von Ebenen), Dice (Bildung von Teilwürfeln), Drill down (eine Ebene tiefer einblenden), Roll up (nächsthöhere Ebene einblenden), und Drill through (die unterste Ebene Einblenden) sind unverzichtbar. Sie genügen aber nicht den Anforderungen, die Kennzahlensysteme für Planungs- und Steuerungszwecke erfüllen müssen.

Wichtige Bedingungen sind:

- Anzahl und Art der möglichen Kennziffern darf nicht beschränkt sein. Der Benutzer muss online neue Kennziffern definieren können. Das System muss ihn dabei durch Aufzeigen bereits vorhandener Kennziffern unterstützen. Eingegebene Definitionsgleichungen müssen interpretativ ausgewertet werden.

- Eine Unterscheidung in Auswertungsmodus und Planungsmodus ist erforderlich.

Im Auswertungsmodus wird durch das System Konsistenz aller Kennziffern mit den Basisdaten, d.h. den Konten der KHBV bzw. den Abrechnungsdaten gemäß 301 SGB V, garantiert. Der Auswertungsmodus dient dazu, den Ist-Zustand anzuzeigen sowie Auffälligkeiten und Abweichungen von den Plandaten deutlich zu machen. Ein Schreiben von Daten ist im Auswertungsmodus vom Grundsatz her nicht möglich. Etwaige Schreiboperationen erfolgen nur temporär. Sie dienen der „what if“- bzw. ,what to do to achieve“-Simulation um Konsequenzen von PlanIst-Abweichungen zu analysieren.

Der Planungsmodus dient dazu, Vorgaben (Plan- bzw. Sollwerte) für einzelne Kennziffern für zukünftige Perioden zu bestimmen. Planungsprozesse erfolgen typischerweise Top-Down, d.h. beginnend mit den aggregierten Spitzenkennziffern werden Vorgaben entlang der Hierarchie nach unten heruntergebrochen. Den nachgelagerten Unternehmensebenen bleibt es dann überlassen, wie sie die Vorgaben umsetzen können. Beispielsweise kann die Forderung, den Gewinn um 10\% $\mathrm{zu}$ erhöhen, wegen der Beziehung, Plan-Gewinn = Plan-Umsatz - Plan-Kosten, durch eine entsprechende Umsatzerhöhung oder Kostenreduktion oder eine Kombination aus beiden erreicht werden. Die Vorgabe eines Plan-Gewinns macht die Gleichung Plan-Gewinn = Plan-Umsatz - Plan-Kosten solange inkonsistent, bis die untergeordneten Kennziffern Plan-Umsatz und Plan-Kosten bestimmt sind. Für jede einzelne Plan-Kennziffer ist folglich der Konsistenzzustand festzuhalten. Mit Abschluss der Planungsphase ist die Plankonsistenz zu überprüfen.

Die weiter oben bereits erwähnten ,what if“" bzw. „what to do to achieve“Simulationen sind ein wichtiges Hilfsmittel zur Unterstützung des Planungsprozesses. 


\subsection{Kennziffernsystem als Aufsatz zu einem Data Warehouse}

Die obige Forderung nach freier Gestaltbarkeit der Kennziffern durch den Benutzer übersteigt die Möglichkeiten der Data Warehouse Technologie, die nur die Kategorien ,Fakt“ und „Dimension“ kennt. Ein „Fakt“ bezieht sich auf einen einzelnen Buchungssatz, also beispielsweise einen Abrechungssatz eines einzelnen Patienten mit einer gesetzlichen Krankenkasse nach § 301 SGB V. Beim Design eines Data Warehouse muss die Faktenbasis explizit bekannt sein.

Die „Dimensionen“ kennzeichnen die möglichen Auswertungen und Aggregationen. In einem Data Warehouse System werden alle Dimensionsausprägungen explizit gespeichert, beispielsweise bei der Dimension „Zeit“ die Zuordnung des Buchungssatzes zu einem bestimmten Tag, einer bestimmten Woche, Monat und Jahr. Oder bei der Dimension „Diagnose“ die Zuordnung zu einer DRG bzw. MDC. Damit müssen alle Dimensionsausprägungen beim Design des Data Warehouse erfasst werden.

Ad hoc gebildete Kennziffern scheiden folglich sowohl als Fakt wie als mögliche Dimension aus. Ein Kennziffernsystem entsprechend den hier vorgestellten Überlegungen kann folglich nur als Aufsatz zu einem bestehenden Data Warehouse realisiert werden. Die Forderung nach freier Gestaltbarkeit der Kennziffern bedingt damit einen erhöhten Implementierungs-Aufwand, da auf Standardsoftware nicht zurückgegriffen werden kann. Dies wird in vielen Fällen, insbesondere in der Einführungsphase eines BSC-Systems nur schwer durchsetzbar sein.

\subsection{Kennziffernsystem als Bestandteil eines Data Warehouse}

Die Alternative besteht darin, auf die freie Gestaltbarkeit bzw. Definierbarkeit der Kennziffern zu verzichten und sich auf fest vorgegebenen Kennziffern zu beschränken. Dies gestattet, die Kennziffer, genauer ihre jeweilige Ausprägung, als „Fakt“ im Sinne der Data-Warehouse Technologie zu modellieren. Nach wie vor sind jedoch die einzelnen Buchungssätze mit „Fakten“ zu identifizieren. Dies führt zu einer typmäßigen Gleichbehandlung der Begriffe „Konto“ und „Kennziffer“. Im Folgenden wird nur der Begriff „Kennziffer“ verwendet, allerdings muss durch eine Statusvariable (in Abbildung 2 die Größe „Art“) festgehalten werden, ob es sich um eine primäre Kennziffer handelt, die von einem Konto des vorgelagerten Buchungssystem in ihren Ausprägungen gefüllt wird und damit faktisch eine Kopie des Kontos ist, oder ob es sich um eine sekundäre Kennziffer handelt, zu deren Definition auf andere primäre oder sekundäre Kennziffern zurückgegriffen wird. 


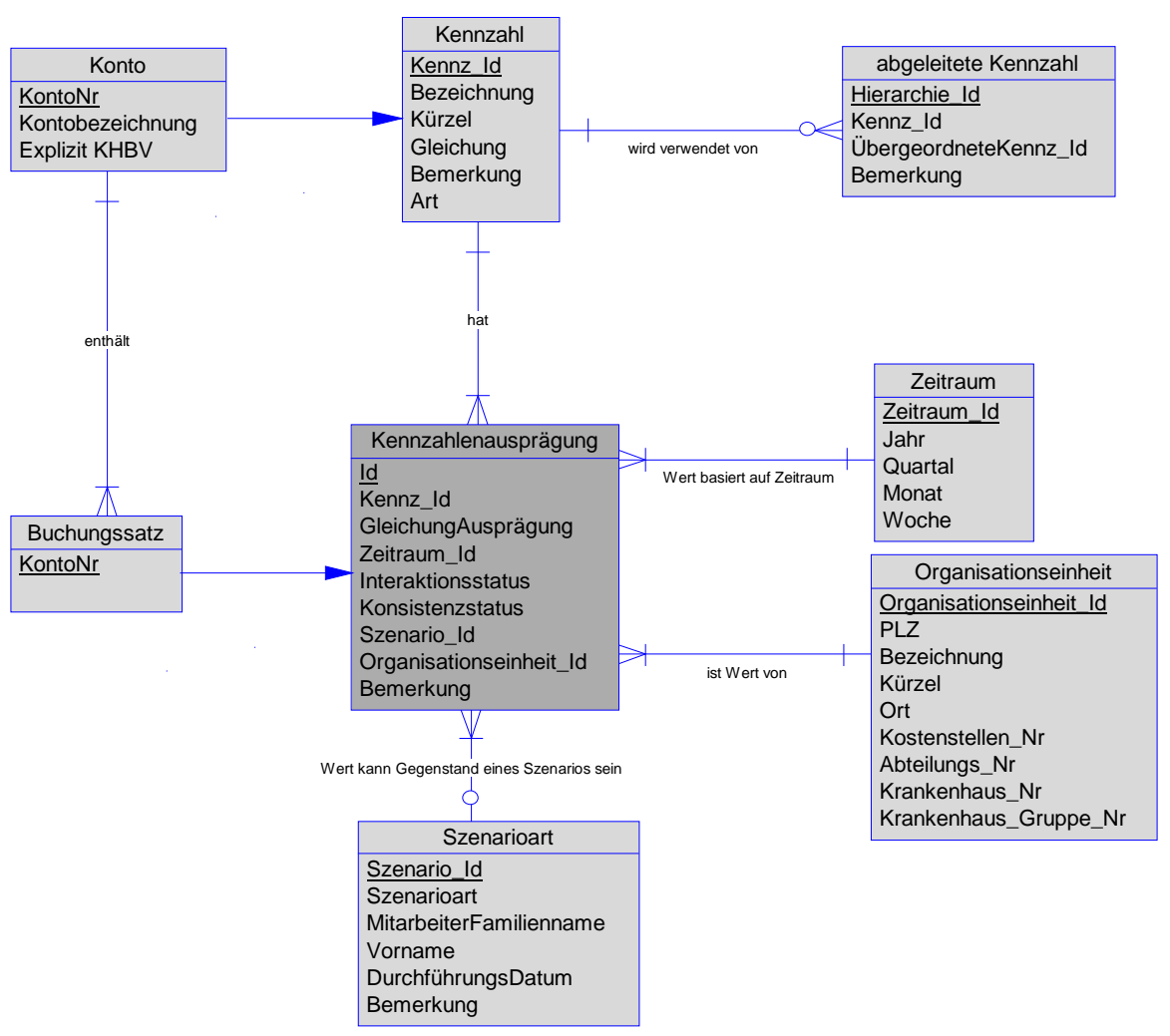

Abbildung 2: Entitity-Relationship-Modell in Form eines Star-Schemas

In Abbildung 2 geben wir die strukturellen Zusammenhänge in Form eines EntityRelationship-Modells an. Hinsichtlich der Repräsentation der Dimensionen „Zeitraum" und „Organisationseinheit" wird das sogenannte Star-Schema verwendet. Wegen der fehlenden Normalisierung hat dieses Schema Nachteile, wenn bezüglich der Dimensionen Änderungen auftreten. Im Beispiel der Abbildung 2 kann sich dies aber nur auf organisatorische Umstrukturierungen beziehen, die vergleichsweise selten vorkommen. Der Vorteil des Star-Schemas gegenüber dem alternativ in der Literatur diskutierten Snowflake-Schema liegt jedoch in der besseren Performance, die für den Alltagsbetrieb große Relevanz hat.

\section{Schlussbemerkung}

Die Notwendigkeit qualitativ hochwertig und gleichzeitig kosteneffizient zu handeln, wird von den Krankenhausverwaltungen sehr deutlich gesehen. Durch die gesetzliche Pflicht, umfangreiche Datensammlungen pro Patient zu erstellen, ver- 
fügen die Krankenhäuser über die erforderlichen Basisinformationen, diesen Zielen gerecht zu werden. Es fehlt zur Zeit an geeigneten Instrumenten, die eine Verdichtung und entscheidungsrelevante Aufbereitung dieser Daten erlauben.

Das hier vorgestellte Konzept zeigt den Weg auf, den die Krankenhäuser beschreiten müssen, wenn sie Erfolg haben wollen. Konkrete Umsetzungsschritte sind in nächster Zeit vorgesehen.

\section{Literatur}

[Bau 01] Baur, A.; Günzel H. (Hrsg.): Data-Warehouse-Systeme. Architektur, Entwicklung, Anwendung, Heidelberg, 2001.

[Böck 01] Böcker, K,; Henke, N.; Krishnan, H.S.; Mansky, T.; Paffrath, D.; Steiners, D.: Diagnosis Related Groups - Grundstein für ein Abrechnungssystem der Krankenhäuser und Krankenkassen. Implikationen und Herausforderungen. In: Die Zukunft des deutschen Gesundheitswesens. Perspektiven und Konzepte, Salfeld, R.; Wettke, J., Hrsg., Berlin, Heidelberg 2001, S. 49-76.

[Haen 01] Haenecke H.; Müller, F.; Nederegger, G.; Thiäner, M.: Faktenbasiertes Management einer Krankenkasse - Führen nach Zahlen. In: Die Zukunft des deutschen Gesundheitswesens. Perspektiven und Konzepte, Salfeld, R.; Wettke, J., Hrsg., Berlin, Heidelberg 2001, S. 111-123.

[Kap 97] Kaplan, R.S.; Norton, D.P.: Balanced Scorecard. Strategien erfolgreich umsetzen. Aus dem Amerikanischen von Horváth P., Kuhn-Würfel B. und Vogelhuber C., Stuttgart 1997.

[Ned 01] Nederegger, G.; zu Putlitz, J.; Thiäner, M.; Wallmann, C.: Zukunft des gesetzlichen Krankenkassenwesens in Deutschland: von der Budgetierung zur wert- und leistungsorientierten Gesundheitsversorgung. In: Die Zukunft des deutschen Gesundheitswesens. Perspektiven und Konzepte, Salfeld, R.; Wettke, J., Hrsg., Berlin, Heidelberg 2001, S. 77-94.

[ThiKr 97] Thiex-Kreye, M.: Management-Informationssysteme auf Basis der OLAPTechnologie. In: Greulich, Thiele, Thiex-Kreye Hrsg., Prozessmanagement im Krankenhaus. Heidelberg, 1997, S. 223-266.

[Vos 99] Vossen G.: Datenbankmodelle, Datenbanksprachen und Datenbankmanagementsysteme. 3. Auflage, München Wien 1999. 